\title{
Architecture and Design of Expert System for Quality of Life Evaluation
}

\author{
Irena ATANASOVA, Jiř́ KŘUPKA \\ Faculty of Economics and Administration, \\ Institute of System Engineering and Informatics, \\ University of Pardubice, Studentská 84, 53210 Pardubice, \\ Czech Republic \\ irena.atanassova@gmail.com, jiri.krupka@upce.cz
}

This article presents an architectural framework of an expert system (ES) in the social area domain and describes the design of the ES, using the shell Expert System for Text Animation (ESTA). The designed system is intended for the evaluation of quality of life $(Q L)$. The development of ES for QL evaluation is a new information technology derived from artificial intelligent research, using ESTA system with application in social area domain. The proposed ES contains knowledge about sets of factors (objective conditions and subjective perception) and indicators, which may be used to QL measure, as followings: equal protection of the law; freedom from discrimination; right to be treated equally without regard to gender, race, language, religion, political beliefs, nationality, socioeconomic status and more; right to privacy; freedom of thought; free choice of employment; right to fair pay; equal pay for equal work; right to vote; right to rest and leisure; right to education. The system is developed in the ESTA, which is Visual Prolog 7.3 Application. The knowledge for the ES will be acquired from domain experts, texts and other related sources.

Keywords: Expert System, ESTA, Quality of Life, Knowledge Base

1 Introduction

In recent decades, considerable efforts have been made to create intellectual and expert programs simulating the professional activity of a man. ESs can be used in those fields of man's activity or at such stages of data processing, where it is difficult to develop and use a single algorithm for solving a complex problem or where one has to use fuzzy data.

In any social system, accumulation and integration of related knowledge and information from many diverse sources play important role. Social specialists and raw experiences are the common sources to provide information that the different stakeholders require for evaluation of quality of life. Social specialists' assistance is not always available when the need arises for their help. In recent years, tools, technologies and applications of information technologies have emerged as efficient and effective measures for the social area specialists. Integration of ES as a powerful tool for the stakeholders of social area has extensive potential. The main concern of the present study is the design of an ES for inter- pretation of knowledge about way of living. The system background starts with the collection of different factors and indicators connecting to QL from social experts, economists and literature. The acquired knowledge is represented to an expert system in Prolog based expert system shell ESTA in order to the knowledge base to be developed. This article presents the conceptual framework of QL. The main goal of this research is to gather and integrate a lot of information for the social area in one place.

\section{Expert Systems in Social Area Domain}

The applications of ES are rapidly increasing. Such applications are very effective in situations when the domain expert is not readily available. In social area, the applications of ESs are mainly found in the fields as the disease diagnosis, the diseases prevention and control, teaching and education, end etc. Many domain specific ESs are being used at different levels.

[20] concerns a class of computing applications, called ESs, which have evolved from the field of artificial intelligence. ES con- 
cepts, potentials and implications are presented along with the issues involved in their development and use. A simple ES, written in BASIC, is included to illustrate the concepts. ES offer great potential for assisting human service professionals in making complex decisions.

„Expert consulting systems in social welfare": [21] describes the origins of ESs and their characteristics, explores the circumstances under which they might be useful in social work, and considers several issues and problems in their application. Although the use of expert consulting systems for planning casework activities is emphasized, the ideas are applicable as well to other areas of social work.

"Teaching Applications of Expert Systems" [14]. Commercial ESs development shells for desk-top computers offers social work educators and trainers a tool for developing computer based classroom and computer laboratory teaching applications. ESs can be used to develop knowledge based applications that provide tutorial support, give and score examinations, automate programmed learning exercises, and teach decision making. Further, many shells are low in cos and have extensive documentation. They are easy to learn thereby allowing teachers to create applications unique to their course needs.

"Formalizing the Social Expert's Knowledge" [4] This ES has as its knowledge base social knowledge and as its inference engine a cognitive model of the way the social expert uses this knowledge. The ES SKI uses the expert's knowledge of social knowledge to explicate implicit information in the protocol and thereby diagnose and correct errors of explication in the novices coding of the protocol. By employing SKI it is possible to increase the level of reliability and replicability in the coded data without having the expert code each verbal protocol and without spending extensive time training the novice. This research is indicative of the way ESs and the findings in artificial intelli- gence can be used in the social sciences.

The main purpose of developing of all those systems is referring to improving the quality of life of people.

\section{Design of the Expert System for Evalua- tion of Quality of Life}

The idea behind creating an ES is that it can enable many people to benefit from the knowledge of one person - the expert. ES simulates the judgment and behavior of a human that has expert knowledge and experience in a particular field. In the design and development of this ES we are using the shell ESTA. ESTA has the explanation facilities of the questions in the knowledge base and for the given advice. ESTA contains the rules represented in its own syntax for its knowledge base. It consists of the inbuilt facilities to write the rules that build the knowledge base.

In ES development, the knowledge base development is the most important part. The quality of an ES depends on knowledge base of the system. Knowledge base development with the help of domain specific expert in this ES is to be developed with ESTA. The process of developing ES, using ESTA, is a multi-step process which aims at developing a domain specific knowledge base. The steps for developing knowledge base in this system are identification of the input problem, gaining knowledge and representation of knowledge into the knowledge base. Here is presented a comprehensive description of each of them. The architecture of the ES is illustrated in Figure 1.

The architecture of a modern ES is based on the clear separation of a system of facts from the inference mechanism formed by two constituent subsystems - the so called knowledge base and inferencing machine [17], [12].

The process of developing knowledge base is:

a) Identifying the input problem

b) Gaining knowledge

c) Representation of knowledge 


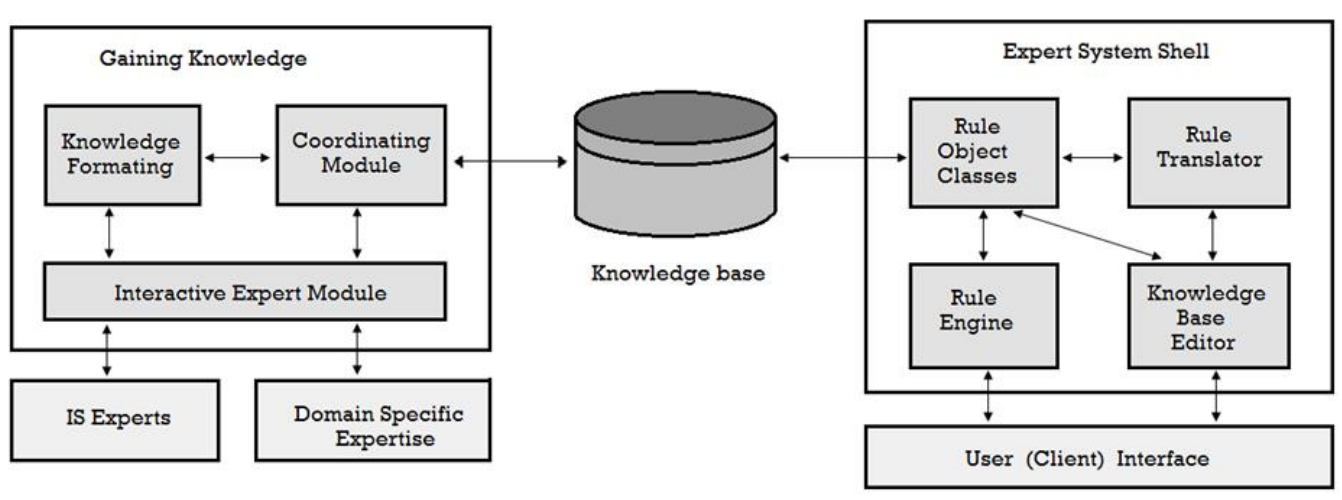

Fig. 1. The ES architecture

\section{Identification of the Input Problem}

To develop an ES, first we need to identify the problem and understand the major characteristics of the problem, which we have to solve in the ES. The input problem for our ES is diverse factors and indicators connecting to social area domain for evaluation of QL.

Since the Lisbon Strategy is considered as a partial failure and the global recession that got started in 2008 is still here, both of them have triggered the understanding for new hallmarks for QL. In terms of the recovery from recession the European Commission has insisted of new interpretations in order to face augmentation of diverse dimensions of human well-being, going beyond the pursuit of the gross domestic product (GDP) growth.

As held by the European Commission Communication "GDP and beyond - Measuring the progress in a changing world" [5], Member States should improve and develop data and indicators in order to complement conventional analyses of economic outputs. In addition, an increasing number of national and international initiatives have been working out with regard to a more detailed analysis of economic and social progress. For example, in June 2007 the European Commission, the OECD, the Organization of Islamic Conference, the United Nations and the World Bank organized a conference on measuring the progress of society in the era of globalization [6]. Also, in 2008 the former French President appointed a commission of experts chaired by John Stiglitz with the end to a report on alternative approaches for measuring both subjective and objective di- mensions of individual well-being [22]. Lastly, in 2010, the United Nation also introduced new measures for gender inequality and for poverty.

Some differences between the "old" and the "new" prospects on QL are considered, and it mostly depends on the constellations in which they were developed. During the 1960s, the attention regarding quality of life was gravitated around the optimistic idea that social monitoring should have rested upon an important instrument for modelling the relentless progress of industrial societies in the age of abundance. On the contrary, the novel debate on the quality of life takes place in the current global economic recession marked by growing inequality and insecurity. That is why the current crisis has remodelled the renewed interest in quality of life mainly in the direction of sustainability. In the new Europe 2020 strategy amidst the others there is also the concept of sustainability - and especially environmental sustainability - which is additionally focused on the alleviation of poverty and deep-seated inequalities in a politicaleconomic context characterized by cuts in public spending and services. According to this view, future generations should not be sacrificed in the name of the willingness for a better life here and now. This indicates a shift from a conceptualization of quality of life as a result of the implacable progress of civilization to the idea of quality of life as a shared and balanced process. Also, it should take into account the impact of policy choices on social and environmental spheres as well as on public budgets. 


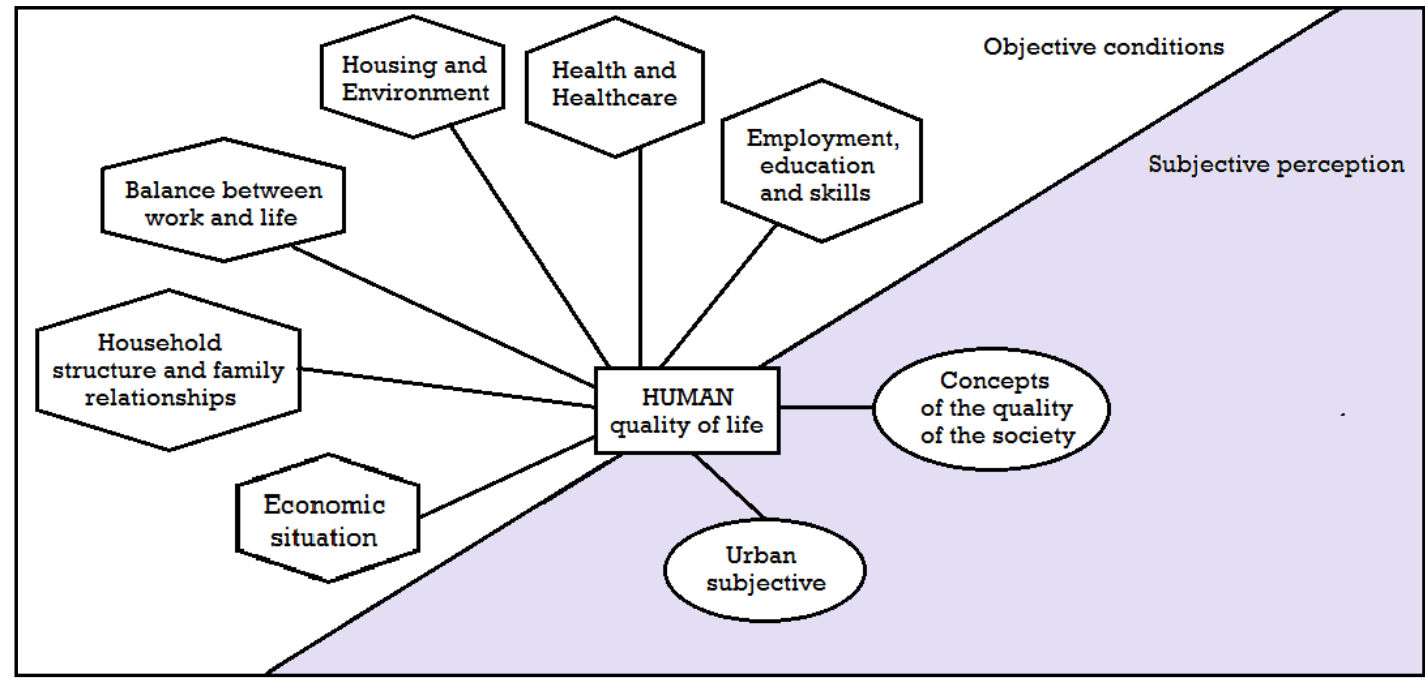

Fig. 2. Domains, affecting the QL

The current model (Figure 2) is based on eight key domains. The first six of them examine objective conditions, the rest analyse subjective perception:

- economic situation: The incomes distribution and degree of privations are very diverse in the different parts of Europe. It is a very important problem, which is referring to building up of one homogeneous European society [8], [10].

Indicators:

- Equal protection of the law;

- Right to vote;

- Right to be treated equally without regard to gender, race, language, religion, political beliefs, nationality, socioeconomic status and more;

- Revenues from environmental taxes;

- Territorial development and utilization of land;

- Frequency of food shopping.

- housing and environment: Asset estate status shows the financial power and long-term reliability. Both the housing conditions and environment are important equally in determining the $\mathrm{QL}$ [7], [13].

Indicators:

- Insufficient space;

- Moisture and leaks;

- Old windows and under;

- Phosphates and nitrates in the water;

- Generation of municipal garbage;

- Presence of smog.
- employment, education and skills: Quality jobs are essential for one and one's family in the battle with the poverty, and therefore they are important for the process of socialization. Creating more quality jobs aiming amplifying the economic competitiveness is naturally the main goal of the policy on employment in EU. Also a kind of policy that is built on gender equality and fosters an ongoing process of learning during the whole life [8].

Indicators:

- Free education;

- Long life education;

- Pressure at work;

- Actual hours worked;

- Job uncertainty;

- Requirements for promotion;

- Duration of work;

- Right to fair pay;

- Equal pay for equal work;

- Freedom from discrimination;

- Freedom of thought;

- Free choice of employment.

- structure of the household and family relationships: The family plays an irreplaceable role in terms of a person to feel secure, wealthy and beloved. There are different roles determined by the way in which men and women act in a certain household [11].

Indicators:

- Time for housework; 
- Support for household (paid, kinship, friendly, neighborhood);

- Good relations in the family;

- Presence of a partner;

- Income.

- balance between work and life: According to Freud, work and love are two main pillars of the human identity, but they have split somewhere in the past. Besides, the family is determined implicitly not only from love but also from work. Hence, there is in the family such correlation: love is work and work is love. However, when you are paid for your work, there is an emotion. In the past, attempts were made to reconcile the family and paid work by means of gender division of labour, but today this model is put into question not only by women but also by men. As a result, there should be striving for a better balance between work and life, and based on exactly this new context [9].

Indicators:

- Social relationships;

- Children at home;

- Additional learning and training;

- Autonomy in work;

- Support from the employer for balance of work - life.

- Right to privacy;

- Right to rest and leisure.

- health and healthcare: Good health is not only important to our sense of wellbeing, but also determines our ability to achieve our goals. Differences in health status of the population in Europe and the quality of health services should be subject to political consideration [8], [23].

Indicators:

- Free healthcare;

- Disease control and prevention;

- Regular immunization;

- Rehabilitation and socialization;

- Extent of free dental services.

- urban subjective: Own assessment of the individual's QL situation is an important factor that can correct or provide more precise picture [1], [19].
Indicators:

- Current life satisfaction;

- People's optimism about their future;

- Expectations.

- concepts of the quality of the society: QL can be compared with two criteria: how attractive for a living is a society and the degree of trust that European citizens have for each other and for their social and political institutions. The social differences are equally important as well [1], [2].

Indicators:

- Trust in others;

- Assessing the quality of social services;

- Alienation in society;

- Despair;

- Uncertainty in their own communities (Tension in society).

The input problem is structured for the system and the expert module recognizes it as a pattern and forwards it for processing evaluation of QL.

\section{Gaining Knowledge (Knowledge Acquisi- tion)}

Gaining process in this expert system will have the following modules - interactive expert module, knowledge formatting and coordinating module for knowledge database.

The domain specific expertise knowledge is acquired by social area experts in the interactive expert module. The acquired knowledge is analyzed and then processed to obtain a best conclusion for the problem. Then the knowledge is transferred to the information system experts to verify for converting into knowledge formatting module. The process is continued until the best conclusion for the problem is obtained. After verification process the knowledge is transferred into expert system program for formatting and representing the knowledge into the knowledge base. Thus, our knowledge acquisition process has been done through a series of interacting sub modules. It is integrated with the coordinating module for creating the knowledge database until the best conclusion is obtained. 
For our system, expert knowledge has been acquired by browsing Internet and reading standard literatures, related to the social area domain. WHO (WHOQOL): "Measuring Quality of Life" [23] defines quality of life as individuals' perception of their position in life. [18], [13] are the papers about QL modelling, where expert knowledge about input problem could be found. In the papers [15] and [16], most of the common problems related to the QL are described. It is useful for the acquisition of knowledge about QL for this ES.

\section{Representation of Knowledge}

Knowledge representation is the last phase of the knowledge base development. In the representation of knowledge into knowledge base, the knowledge acquired from knowledge acquisition process is represented into structured form. There are many approaches for representing knowledge into the knowledge base. Such representation in ESTA is the rule based representation in logical paradigm of simple IF - THEN rules in backward or forward chaining. Rules consist of two parts: a premise (IF) and an inference (THEN), with each of them consisting, in turn, of a combination of statements of a lower detailed level.

In general, an ES is, typically, composed of two major components, the Knowledge base and the ES shell. Some scientists believe that such a division is quite evolutionary [12], because the knowledge is a qualitatively new form of information and is different from the data used in the traditional programming. In fact, knowledge is one of the possible types of the data and that the dialogue program for data processing, the so called inferencing machine, is the implementation of the deductive algorithms.

\section{Expert System $\rightarrow$ Shell $($ ESTA $)+$ Knowledge Base}

The knowledge base is a collection of rules encoded as metadata in a file system, or more often in a relational database. An essential feature of the expert system is its ability to complement the data base with new information obtained from experts in the process of their dialogue. First, the necessity of complementing the knowledge base with new facts is analyzed; i.e., it is checked whether the fact to be introduced into the knowledge base is really new and whether it is consistent with the other facts of the base. The subsystem of explanations provides the dialogue with a user. The main goal of this subsystem is to make the performance of the program understandable to the user.

The expert system shell is a problemindependent component housing facilities for creating, editing, and executing rules. The shell portion includes software modules whose purpose is to: process requests for service from system users; support the creation and modification of business rules; translate business rules into machine-readable forms; execute business rules; provide low-level support to ES components.

\section{User (Client) Interface}

The user (client) interface processes requests for service from system-users and from application layer components. Client interface logic routes these requests to an appropriate shell program unit. For example, when a subject matter expert wishes to create or edit a rule, they use the client interface to dispatch the knowledge base editor. Other service requests might schedule a rule, or a group of rules, for execution by the rule engine.

\section{Conclusions}

We are in the process of development of an expert system, which will be developed in ESTA Application. It is designed for assisting in the interpretation of quality of life and providing the assessment for social life. In this article is presented the architecture and design of an expert system for evaluation of quality of life.

Such system is mainly useful for those users who are not specialists in social area and could not count on that kind of type of specialists in their research work for supporting them in solving problems in the field of interpretation of quality of life. 
The presented architecture is an integrated system with interactive user interface, control and coordinating units, expert system shells, and structured knowledge representations. The design of this expert system considers the dynamic structuring of knowledge representations and rule applier.

\section{Acknowledgement}

This work was supported by the Project No. CZ.1.07/2.3.00/30.0021 - 'Enhancement of R\&D Pools of Excellence at the University of Pardubice', Ministry of Education, Youth and Sports of the Czech Republic and European Social Fund,

\section{References}

[1] J. Alber and T. Fahey, "Perceptions of living conditions in an enlarged Europe, European Foundation for the Improvement of Living and Working Conditions, Office for Official" Publications of the European Communities, Luxembourg, 2004.

[2] A. Alesina, E. La Ferrara, "Who trusts others?", Journal of Public Economics 85 (2002)

207-234, www.elsevier.com/locate /econbase.

[3] Association Quality of Life, 2013, http://qualityoflife.bg/

[4] K. Carley, "Formalizing the Social Expert's Knowledge". Carnegie-Mellon University, 2012, doi: $10.1177 / 0049124188017002003$, Sociological Methods Research, November 1988 vol. 17, no. 2, pp. 165-232, http://smr.sagepub.com/content/17/2/165. abstract

[5] European Commission, 2009: Communication from the Commission to the Council and the European Parliament - GDP and beyond: measuring progress in a changing world, COM/2009/0433 final, Brussels: Brussels: retrievable at http://www.beyond-gdp.eu/.

[6] European Commission, 2009: Summary notes from the Beyond GDP conference. Highlights from the presentations and the discussion. Brussels: retrievable at http://www.beyond-gdp.eu/.
[7] European Commission, 2002, The life of women and men in Europe: A statistical portrait, Data 1980-2000, Eurostat. Theme 3. Population and social conditions, Luxembourg, Office for Official Publications of the European Communities, 2002b.

[8] Eurofound, (2013), European Foundation for the Improvement of Living and Working Conditions, 2012, Annual work programme 2012,

http://www.eurofound.europa.eu/pubdoc s/2012/03/en/1/EF1203EN.pdf.

[9] Eurofound (project no. 0272), 2013, European Foundation for the Improvement of Living and Working Conditions, European survey on working time and worklife balance, http://www.eurofound. europa.eu/areas/worklifebalance/eswt.htm

[10] Eurofound (project no. 0310), 2013, European Foundation for the Improvement of Living and Working Conditions, Integration of migrants and access to social rights: role of local authorities, http://www.eurofound.europa.eu/areas/w orklifebalance/eswt.htm

[11] European Parliament, 2004, Report on reconciling professional, family and private lives, Brussels: Committee on Women's Rights and Equal Opportunities (A50092/2004), 2004a.

[12] R. Forsyth, "Expert Systems. Principles and Case Studies", Ed. (Chapman, London, 1986; Radio i Svyaz', Moscow, 1987), p. 51.

[13] M. Kasparova, J. Krupka, P. Jirava, "Approaches to Air Quality Assessment in Locality of the Pardubice Region", Book of proceedings of the $5^{\text {th }}$ International Conference EMAN 2009.

[14] M. J. Kelly, "Teaching Applications of Expert Systems", Journal of Teaching in Social Work, Volume 9, Issue 1-2, 1994, pages 37-47.

[15] J. Krupka, M. Kasparova, J. Mandys, P. Jirava, "Quality of Life Modelling on the basis of Qualitative and Quantitative Data”. In: Y. Holtzman (Eds.) Advanced Topics in Applied Operations Management, InTech, Rijeka, Croatia, 2012, pp. 
133-156 s., ISBN 978-953-51-0345-5, http://www.intechopen.com/books/advan ced-topics-in-applied-operationsmanagement

[16] J. Krupka, J. Mandys, M. Kasparova, P. Jirava, "Approaches for the Comparison of the Quality of Life Investigation", Book of proceedings of the $5^{\text {th }}$ International Conference EMAN 2009.

[17] PerfectLogic, 2013, http://www.perfectlogic.com/articles/AI/ ExpertSystems/ExpertSystems.html

[18] R. Provaznikova, P. Petr, J. Krupka, 2010, "Analysis of the Regions Based on Multidimensional Methods", TILTAI Vol. 40.

[19] C. Rocke, M. E. Lachman, "Perceived Trajectories of Life Satisfaction Across Past, Present, and Future: Profiles and Correlates of Subjective Change in Young, Middle-Aged, and Older Adults",
Psychology and Aging, 2008, Vol. 23, No. 4, 833-847, Copyright 2008 by the American Psychological Association.

[20] D. Schoech, "Artificial Intelligence for Professional Decisions, Computers in Human Services", Vol. 1, Issue 1, 1985, pp 81-115.

[21] J. R. Schuerman, "Social Work Research Abstracts", 198723 (3): 1418.doi: 10.1093/swra/23.3.14, Oxford University press.

[22] J. E. Stiglitz, A.K. Sen, J. P. Fitoussi, 2009, "Report by the Commission on the measurement of economic and social progress". Available at: http://www.stiglitz-senfitoussi.fr/en/index.htm.

[23] WHO, 2013, World Health Organization, http://www.who.int/en/

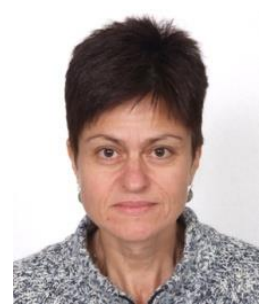

Irena ATANASOVA graduated the Faculty of Mathematics and Nature Sciences of South-West University "Neofit Rilski" - Blagoevgrad, Bulgaria in 1995. She held Ms diploma in Mathematics, Informatics and Management and she joined the staff of the South-West University - senior assistant professor. In 2011 she finished the doctoral thesis in the field of Informatics (Artificial Intelligence). She is the author of more than 12 journal articles in the field of modal logics and knowledge modeling. Her work focuses on the process of knowledge engineering. Currently, she is working as a senior researcher at the University of Pardubice

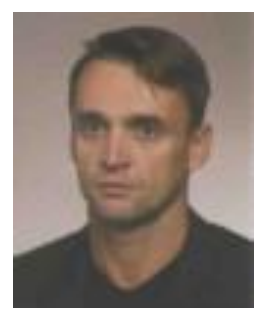

Jiř́ KŘUPKA graduated from the Military Technical University in Liptovský Mikuláš in 1985. From 1985 till 1990 he worked in the Department of Technical Support System's and Automation in the Air Defense. From 1990 till 2004 he worked as a lecturer, a senior lecturer, and vice-dean for education at the Faculty of Air Defense at the Military Academy in Liptovský Mikuláš. There he finished his doctoral thesis in 1995 and habilitated in 1997. Since 2004 he is working as associated professor and head of Institute of System Engineering and Informatics, Faculty of Economics and Administration, University of Pardubice. Assoc. Prof. Křupka has published parts of book and a number of papers concerning with fuzzy decision, fuzzy control, case based reasoning, and rough set theory. Nowadays he is focusing on modelling of environmental and social systems. 